\title{
Is Zero Void? Attentional Mechanism of Hidden-zero Effect in Risky
}

\section{Decision-making}

LEI ZHOU ${ }^{1,3 \dagger}$, LEI ZHANG ${ }^{4,6 \dagger}$, YIN SU ${ }^{5}$, and ZHU-YUAN LIANG ${ }^{1,2^{*}}$

${ }^{1}$ CAS Key Laboratory of Behavioral Science, Institute of Psychology, Beijing 100101, China

${ }^{2}$ Department of Psychology, University of Chinese Academy of Sciences, Beijing 100049, China

${ }^{3}$ Management School, Jinan University, Guangzhou 510632, China

${ }^{4}$ Institute for Systems Neuroscience, University Medical Center HamburgEppendorf, Hamburg 20246, Germany

${ }^{5}$ User Research Group of Alimama Business Unit, Alibaba Group, Beijing 100102, China

${ }^{6}$ Neuropsychopharmacology and Biopsychology Unit, Department of Basic Psychological Research and Research Methods, Faculty of Psychology, University of Vienna, Vienna, 1010, Austria

${ }^{\dagger}$ These authors contributed equally to this work.

Author note

Author contributions: Conceptualization: Z-Y.L. \& Y.S.; Methodology: L.Zhou, Z-Y.L. \& Y.S.; Software: L.Zhou; Data collection \& curation: L.Zhou \& Y.S.; Formal analyses: L.Zhou; Computational Modeling: L. Zhang; Writing: L.Zhou, L.Zhang \& ZY.L.; Supervision: Z-Y.L.

${ }^{*}$ Correspondence to: Dr. Zhu-Yuan Liang, Institute of Psychology, Chinese Academy of Sciences, Beijing 100101, China. E-mail: liangzy@psych.ac.cn. 
HIDDEN-ZERO EFFECT IN RISKY DECISION-MAKING

\section{Is Zero Void? Attentional Mechanism of Hidden-zero Effect in Risky}

\author{
Decision-making
}

The frame of risky choice could alter and shape an individual's risky preferences. Within an option of typical risky choice, that is, " $p \%$ chance to win $\$ Q$," naturally embedded a hidden-zero outcome, namely, “ $(1-p) \%$ chance to win $\$ 0$.” Despite its pervasive existence, there is insufficient evidence of the existence or a cognitive mechanism of the hidden-zero effect in risky choice. To this end, we proposed an attentional based risk-aversion model for behavior and process level to interpret the mechanism of the hidden-zero effect. We presented participants' explicit or hiddenzero outcomes in pairs of certain versus risky options and measured their choice preferences and eye-movement characteristics. We observed that participants were less risk avoidant in the explicit-zero condition than in the hidden-zero condition, and a descriptive attentional bias shifted this preference to favor certain options in the hidden-zero condition (Study 1). We further combined the eye-tracking data with hierarchical Bayesian modeling (Study 2). We observed that a model combining behavioral and process attention provided better predictions regarding participants' preference. When presenting zero outcomes, an empirical attentional bias integrating eye-movement features indicated that attention plays a central role to alter the attention allocation and consequent choice preference from certainty options to risky options in risky decision-making. These findings highlight the potential mechanism of the hidden-zero effect in risk decision-making on cognitive and computational levels. modeling; risky decision-making 
3 For example, to simplify decision-making, one of the most common and classical

4 forms of risky choices goes on in our daily life, and risky decision research, is the

5 choice preference between bioptional alternatives: one risky and one certain option

6 with a non-zero outcome and its corresponding probability (e.g., 70\% chance to gain

7 \$100). Crucially, these partially described contexts naturally neglect the co-existed-

8 albeit hidden-zero — outcomes (e.g., 30\% chance to gain \$0). Several studies have

9 indicated that merely changing the completeness of presentations (i.e., fully or

10 partially describing options) while keeping the absolute value of options intact may

11 alter individuals' preference in the framing effect of risky decision-making

12 (Kühberger \& Tanner, 2010; Schulte-Mecklenbeck \& Kühberger, 2014) and

13 intertemporal choice (Magen, Dweck, \& Gross, 2008; Read, Olivola, \& Hardisty,

14 2016). However, according to our review of the literature, few studies have

15 investigated the impacts of the co-existing but hidden zero outcomes on risky choice

16 and the potential mechanisms of the shifted choice preference. Therefore, this study

17 aimed to thoroughly uncover whether explicitly presenting zero outcomes alters

18 individuals' choice preferences in risky decision-making and reveal its underlying

19 mechanism using computational modeling.

\subsection{Zero outcomes in classic models of risky decision-making}

Whether the existence of zero outcomes alters choice preference in risky choice is essential to test classic computation-based decision models. Classic risky decisionmaking models based on mathematical computation are by default silent to the hidden information in risky options. These models share a common assumption that rational decision-makers would compute the values for each option through a weighting and summing process and select the option with the highest value. Examples are the 
HIDDEN-ZERO EFFECT IN RISKY DECISION-MAKING

1 classic Expected Value (EV) theory (Pascal, 1670) and its successors, Expected Utility

2 theory (EU; von Neumann \& Morgenstern, 1947), Prospect theory (PT; Kahneman \&

3 Tversky, 1979), and Cumulative Prospect theory (CPT; Tversky \& Kahneman, 1992).

4 In all those models, the EV or utility of an option with a zero outcome (or zero

5 probability) is always equal to zero; therefore, the zero outcomes do not necessarily

6 affect the final decision.

\subsection{Effects of zero outcomes on decision-making}

Contrary to the general neglect of zero outcomes in classic computation-based

9 decision models, recently, an increasing number of studies have shown that zero

10 outcomes of options affect choice preference in a variety of types of decision-making.

11 For example, Magen and colleagues (2008) first reported the hidden-zero effect in

12 intertemporal choice. In their study, compared with a silencing of the zero outcome,

13 explicitly referring to the zero outcome in each alternative (e.g., "Would you prefer

14 [A] \$5 today and \$0 in 26 days OR [B] \$0 today and \$6.20 in 26 days?") decreases

15 participants' willingness to choose impatient choices (i.e., “To receive $\$ 5$ today").

16 Similarly, in consumer choice, consumers have difficulties making decisions when

17 one of the product attributes is zero, namely, the zero-comparison effect (Palmeira,

18 2011). In his view, zero indicates an absence of a reference point to evaluate its

19 meaning; thus, individuals do not necessarily know how good or bad zero is and

20 would not react strongly to options with zero outcomes.

21 In the risky choice field, however, the possible impact of zero outcomes has not

22 gained sufficient attention compared with intertemporal choice. The seminal research

23 by Kühberger and his colleagues has indicated that hiding or presenting specific

24 components of choice options eliminates or enhances the classic framing effect

25 (Kühberger \& Tanner, 2010, 2010; Schulte-Mecklenbeck \& Kühberger, 2014). For

26 example, hiding the zero outcome of a risky option (e.g., $2 / 3$ probability that nobody

27 will be saved) would eliminate the framing effect, whereas presenting only the zero 


\section{HIDDEN-ZERO EFFECT IN RISKY DECISION-MAKING}

1 component intensifies the framing effect (Kühberger \& Tanner, 2010). Kühberger

2 explained these results based on the fuzzy-trace theory: Individuals reduce all

3 quantitative information (e.g., 1/3 probability that 600 individuals will be saved/dead)

4 into a qualitative gist (some individuals will be saved/dead) and choose the alternative

5 that leads to more lives saved. Zhang and Slovic (2019) reported a zero effect in the

6 loss frame of life-saving decisions: Presenting a zero outcome pushes an individual to

7 choose based on the EV of options. Even when the first gamble offered a larger

8 expected loss, participants had strong preferences for an option with zero outcome

9 (50-50 chance that 8 or no one will die) than that without zero outcome (50-50

10 chance that 4 or 3 will die). These findings imply the hidden-zero effect in risky

11 choice may also exist. However, no direct evidence is available regarding the

12 existence and cognitive mechanism of hidden-zero effect in risky decision-making.

\section{$13 \quad 1.3$ Attention process underlining the hidden-zero effect}

14 Attention is one of the most important aspects and crucial mechanisms

15 underlining decision-making processes (Brandstätter and Körner, 2014; Krajbich, Lu,

16 Camerer, \& Rangel, 2012; Krajbich \& Rangel, 2011). Attention could influence

17 behavioral choices (i.e., choice bias) and decision processes (i.e., attentional bias).

18 Individuals are more likely to choose the options with more attention or been last

19 fixed (Brandstätter and Körner, 2014; Krajbich, Lu, Camerer, \& Rangel, 2012;

20 Krajbich \& Rangel, 2011). Attention may also modulate the speed of value integration

21 in the relative decision value evolving, as assumed by the attentional drift-diffusion

22 model (aDDM) (Krajbich et al., 2010; Krajbich \& Rangel, 2011). This effect of

23 attention to decision-making has gained solid direct evidence. For instance,

24 manipulating attention can alter the preference in intertemporal choice and moral

25 decisions (Fisher \& Rangel, 2013; Pärnamets et al., 2015); shifting the attention of the

26 message recipient to the complementary frame would reduce or even eliminate

27 attribute-framing bias, that is, the tendency to evaluate objects differently when 
HIDDEN-ZERO EFFECT IN RISKY DECISION-MAKING

1 objects are framed positively or negatively (Kreiner \& Gamliel, 2018).

2 Parallel with this line of research, several exploratory studies have proposed that

3 attention may underline the cognitive process of the hidden-zero effect in

4 intertemporal decision-making. For example, Radu and colleagues (2011) examined

5 the "temporal attention hypothesis" and observed that explicitly presenting zero

6 outcomes shifted participants' attention away from immediate options and toward

7 more distant options, increasing participants' patient choice. Additionally, when

8 explicitly highlighting the zero outcomes, participants allocated more attention to later

9 but larger (LL) options than sooner but smaller (SS) options (Read et al., 2016).

10 However, this role of attention in the hidden-zero effect has not been investigated in

11 risky choices.

$12 \quad 1.4$ Attentional risk-aversion model of hidden-zero effect in risky choices

13 Due the aforementioned deficiencies of the EU-based model family on zero

14 options (zero outcome or zero probability), we developed a series of attentional risk-

15 aversion (aRA) models to capture the cognitive processes in the hidden-zero effect,

16 with which, we could predict choice preference in risky choices. Given that we were

17 interested in the asymmetry preference in the hidden-zero effect, we focused on a PT-

18 based risk-aversion model (RA) as a baseline model for its flexibility and

19 adaptiveness: RA has been shown to capture the potential behavioral asymmetry

20 choice preferences in loss aversion (e.g., Sokol-Hessner et al., 2009; Sokol-Hessner,

21 Camerer, \& Phelps, 2012), which outperformed EU-based models. Furthermore, most

22 of the decision models in the literature, at least to some extent, have not yet been

23 informed by empirically valuable source evidence that directly examines the

24 underlying processes; as Marewski ingeminated, "models ought to be fully specified

25 so that they are able to not only post hoc describe but also a priori predict behavior

26 and they should make detailed predictions about cognitive processes rather than just

27 predicting decisional outcomes" (Marewski \& Bröder, 2018). 


\section{HIDDEN-ZERO EFFECT IN RISKY DECISION-MAKING}

To bridge this gap, we introduced an attentional bias into the RA model and developed several variants of the RA model in order to improve the model performance regarding explaining the cognitive mechanism of risky decision-making in the hidden-zero effect. Notably, our aim is to compare models and progressively develop models from their basic form to include attentional bias and combine process data (e.g., eye movement).

\subsubsection{Attentional risk-aversion model on the behavioral level}

To compare the effectiveness of the attentional bias in the hidden-zero and explicit-zero condition, we developed two RA model variants for the hidden-zero condition (RA and aRA) and two RA-p $\lambda$ model variants (see below) for the explicitzero condition (RA- $p \lambda$ and aRA- $p \lambda)$.

In all models, the subjective value of the certain option follows the value function:

$$
V_{c}=100 \% \times X^{p}=X^{p}
$$

where $V c$ and $X$ represent subjective value and the objective payoff of certain options, respectively. The $\rho$ parameter $(0<=\rho<=2)$ governs the size of the subjective value given the sure payoff.

For the value of the risky option, because there were only zero payoffs rather than losses in our experimental settings, the direct formulation based on the $R A$ model is to assign a " 0 " at the loss position.

$$
V_{R}=p \times X^{p}=p \times 0=0
$$

In the explicit-zero condition, however, we assume that the risk (i.e., probability) of receiving the " 0 " payoff may alter participants' choice preference when deciding between the risky option and the certain option. Thus, we introduced an $R A-p \lambda$ model and defined the value function of the risky option as follows:

$$
V_{R}=p \times X^{p}-\lambda(1-p)
$$

where $p$ is the probability that is associated with the risky payoff, and the $\lambda$ parameter 
HIDDEN-ZERO EFFECT IN RISKY DECISION-MAKING

$1 \quad(-5<=\lambda<=5)$ determines the effect of the probability of obtaining the zero payoff.

2 After we compute the values of both options, we convert the values into choice

3 probabilities by using the Softmax action selection rule (Sutton \& Barto, 1998). The

4 probability of choosing the risky option is determined by

$$
p(\mathrm{R})=\frac{1}{1+e^{\tau\left(-\left(V_{R}-V_{C}\right)\right)}}
$$

6 where the $\tau$ parameter $(0<=\tau<=5)$ is the inverse Softmax temperature that controls

7 the stochasticity of participants' choice. More important, we have now hypothesized

8 that participants' attention bias is crucial in the current task, which introduced a

9 descriptive attentional bias, $\theta$, which resulted in the aRA model and the aRA-p $\lambda$

10 model, respectively. As such, we have

$$
p(\mathrm{R})=\frac{1}{1+e^{\tau\left(-\left(V_{R}-V_{C}+\theta\right)\right)}}
$$

To avoid the non-identifiable behavioral in the mode fitting caused by the presence of $\theta$, given the product between $\tau$ and $\theta$, we then rearrange the formulation and obtain a regression-like component in the Softmax:

$$
p(\mathrm{R})=\frac{1}{1+e^{\beta 0+\beta 1\left(-\left(V_{R}-V_{C}\right)\right)}}
$$

Here, $\beta_{0}$ and $\beta_{1}$ depict the effect of the descriptive attentional bias and subjective

17 value, respectively (See Table A3 for the summary of models).

\subsubsection{Attentional risk-aversion model at the process level}

As aforementioned, to include process measurement as a crucial building block in our cognitive models, we incorporated selection bias (SB) score derived from eyemovement data into our models to account for trial-by-trial attentional processes. The SB scores represent a direct measure of overt attention to a specific element (FrancoWatkins, Mattson, \& Jackson, 2016), that is, the attention allocation pattern across different options and outcome levels. This features thus enables us to construct an empirical attentional bias (relative to descriptive attentional bias introduced in Study 
HIDDEN-ZERO EFFECT IN RISKY DECISION-MAKING

$$
\beta_{0}=\beta_{0}{ }^{\prime}+\beta_{0 \mathrm{SB}} \times S B_{X}
$$

where $S B \mathrm{X}$ is a type of $S B$ score, and $\beta_{0 \mathrm{SB}}$ quantifies the effect of the corresponding $S B$ score. Accordingly, by applying this new $\beta_{0}$ to Eq. 6 , we rearranged the new parameters and the $p(\mathrm{R})$ now becomes

$$
p(\mathrm{R})=\frac{1}{1+e^{\beta 0+\beta 1 \mathrm{SB}+\beta 2\left(-\left(V_{R}-V_{C}\right)\right)}}
$$

This parameter reconstruction has at least two advantages. First, as we have calculated the SB score for every trial, we can probe into the attentional bias on a trial-by-trial basis and track the within-trial variance. Second, because the SB score is obtained from a separate modality (i.e., eye movement) that implies trace processes, a benefit could be realized in the development and interpretation of our cognitive model (See Table A3 for the summary of models in Study 2).

\subsection{Scope and hypotheses of this study}

In this study, we explored the existence of the hidden-zero effect in risky decision-making and developed computational models that utilize an attentional $R A$ model as their core to quantify the attentional process and the corresponding choice preference in the hidden-zero effect. In Study 1, we used a questionnaire and hierarchical Bayesian modeling to first elucidate the existence of the hidden-zero effect in risk decision-making and then assess how attentional bias is implemented to guide an individual's choice preference in the hidden-zero condition and explicit-zero condition (Study 1).

In Study 2, we focused on the trace process (using eye-tracking) of the hiddenzero effect and accommodated the process data into our models by synthesizing the evidence of behavioral readouts and process dynamics. We explored the connection between SB and the hidden-zero effect on the process level and examined their performance.

Taken together, we proposed three hypotheses:

Hypothesis 1 (H1): The hidden-zero effect exists in risky decision-making. 


\section{HIDDEN-ZERO EFFECT IN RISKY DECISION-MAKING}

1 Namely, explicitly presenting zero outcomes reduces the proportion of choosing

2 certain options.

Hypothesis $2(\mathrm{H} 2)$ : The reduced tendency to decide on certain options might be accounted for by an attentional bias at the valuation level, with explicitly presenting zero outcomes alters the bias such that risky options are more preferred than certain options. risky decision-making, which would be reflected by the increased model performance by accommodating eye-movement data that track the trial-by-trial attention feature.

\section{Study 1}

$11 \quad$ 2.1. Method

\subsubsection{Participants}

The sample was 137 undergraduate or postgraduate students $\left(\right.$ female $=78, M_{\text {age }}=$

15 Academy of Sciences. Each participant was paid CNY 30 (CNY $1 \approx$ USD 0.15$)$ in

16 cash for participation. All participants provided oral consent prior to the study.

\subsubsection{Materials and procedure}

Participants were instructed to fill in a paper-based questionnaire that comprised

20 pair included one certain option (e.g., 100\% probability of receiving CNY 98, outcome

21 value range: CNY 90-99) and one risky option (e.g., 27\% probability of receiving CNY

22369 , outcome value range: CNY 105-1,828). The probability of the risky option was

23 either low $(5 \%-30 \%)$ or high $(70 \%-95 \%)$. Both options in each pair had approximately

24 equivalent EVs (EV difference range: 0-5.5). 


\section{HIDDEN-ZERO EFFECT IN RISKY DECISION-MAKING}

explicit-zero $(n=63)$ conditions. In the hidden-zero condition, both options had only one outcome (e.g., CNY 98 and CNY 369); thereafter, neglecting the zero outcomes in risky choices is as in classical risky choice tasks. In the explicit-zero condition, both options had two outcomes: one outcome was the same as in the hidden-zero condition, and the other explicitly presented the former's corresponding zero outcomes (e.g., $27 \%$ probability of receiving CNY 369 and 73\% probability of receiving CNY 0; see Appendix for more details).

\subsubsection{Model fitting and model selection}

We adopted the "hBayesDM" package (Ahn, Haines, \& Zhang, 2017) to fit all aforementioned candidate models using the Hierarchical Bayesian Analysis (HBA; Gelman et al., 2014), which uses a Markov Chain Monte Carlo (MCMC) sampling scheme to perform full Bayesian inference and obtain the actual posterior distribution. We employed HBA rather than maximum likelihood estimation (MLE) because HBA could account for population-level generality and individual difference and provide a much more stable and accurate estimate compared with MLE (Ahn, Krawitz, Kim, Busemeyer, \& Brown, 2011).

By following the approach in "hBayesDM" package, we applied weakly informative priors (Gelman et al., 2014) and assumed the individual-level $\theta$ (denoting a generic parameter) was drawn from a group-level normal distribution: $\theta \sim$ Normal $\left(\mu_{\theta}, \sigma_{\theta}\right)$. In HBA, all group-level parameters and individual-level parameters are simultaneously estimated through the Bayes rule by incorporating behavioral data. We fit each candidate model with four independent MCMC chains using 1,000 iterations after 1,000 iterations for the initial algorithm warmup per chain, which resulted in 4,000 valid posterior samples. Convergence of the MCMC chains was assessed visually (from the trace plot) and through the Gelman-Rubin R-hat Statistics (Gelman \& Rubin, 1992). R-hat values of all parameters are close to 1.0 (at most, smaller than 1.1 , in this study), which indicates adequate convergence. 


\section{HIDDEN-ZERO EFFECT IN RISKY DECISION-MAKING}

To compare the effectiveness of the attentional bias in the hidden-zero and the explicit-zero conditions, we tested two RA model variants (RA and aRA) as candidate models for the hidden-zero condition and two RA- $p \lambda$ model variants (RA- $p \lambda$ and aRA- $p \lambda)$ as candidate models for the explicit-zero condition.

For model comparison, we computed the widely applicable information criterion (WAIC) score per candidate model (Vehtari, Gelman, \& Gabry, 2017). The WAIC score provides an estimation of out-of-sample predictive accuracy in a fully Bayesian manner and is more reliable than the traditional point-estimate information criterion (e.g., AIC). A lower WAIC score indicates a better out-of-sample prediction accuracy of the candidate model. A difference score of 10 on the information criterion scale is considered decisive (Burnham \& Anderson, 2004). Therefore, we selected the model with the lowest WAIC as the winning model for subsequent analysis.

\subsection{Results}

Sixteen option pairs $(0.19 \%)$ were excluded for the analysis of Studyl because of null response, resulting in 8,615 valid responses.

\subsubsection{Choice preference}

We calculated the proportion of choosing certain options as the dependent variable and used binary logistic regression implemented in a generalized linear mixed model to predict participants' choices. We took the condition (hidden-/explicitzero) and the probability of the risky option and their interaction as fixed effects predictors, and Subject ID as the random effects (Table A1). The effect of condition was significant $(\beta=-.93, p<.001$, odds ratio $=.39,95 \% \mathrm{CI}=[.29, .54]$, Figure 1$)$, indicating that the presentation of zero outcome negatively predicted individuals' preference for a certain option. These results suggest that presenting the zero outcome prompted individuals to decide more on risky options, despite that both options were approximately equivalent. Hence, these data have demonstrated the existence of the hidden-zero effect in risky decision-making (supporting $\mathrm{H}_{1}$ ). Considering that the 
HIDDEN-ZERO EFFECT IN RISKY DECISION-MAKING

1 proportion of choosing a certain option decreased from $67 \%$ to approximately $50 \%$

2 (Fig. 1), we could infer that the explicitly presented zero decreases the level of risk

3 avoidance.

\section{$============$ Insert Figure 1 about here $============$}

\subsubsection{Model estimate and model comparison}

In the hidden-zero condition, to test hypothesis $\mathrm{H} 2$, we estimated the RA model (M11) and the aRA model (M12) and performed a formal Bayesian model comparison.

M11 contains a subjective value sensitivity $(\rho)$ and a Softmax temperature parameter ( $\tau$; equivalent to $\beta\left(\mathrm{V}_{\mathrm{R}}-\mathrm{V}_{\mathrm{C}}\right)$ ). Adding a descriptive attentional bias term (i.e., $\beta_{0}$ in M12) improved the model performance compared with the RA model, as quantified by the model evidence (WAIC; Fig. 2A). By comparing the winning model's posterior prediction with the actual behavioral data, our aRA model was capable of capturing the behavioral pattern (Fig. $2 \mathrm{C}$ ). Specifically, $\beta_{0}$ in the hiddenzero condition was credibly below zero (mean $=-0.98,95 \%$ highest density interval, HDI $=[-1.37-0.58]$; Fig. 2B), suggesting that in the hidden-zero condition, participants maintained an SB toward certain options (the more negative $\beta$ was, the more likely participants preferred the certain option), and only value differences that were sufficient large to overcome this bias were able to drive participants' preference toward risky options.

Similarly, in the explicit-zero condition, we tested the RA- $p \lambda$ model (M21) and the aRA- $p \lambda$ model (M22).

The Bayesian model comparison showed that the bias term parameter $\left(\beta_{0}\right)$ had no effect on the model estimate (Fig. 2D) and the winning model is the RA- $p \lambda$ model (M21). Despite the model comparison, either model provided a sizable match between model-generated predictions and the actual data (Fig. 2D). This is likely because of the relatively noisy choice behavior of participants in the explicit condition. Out of 63 


\section{HIDDEN-ZERO EFFECT IN RISKY DECISION-MAKING}

1 trials, participants made $31.78 \pm 2.58(\approx 50 \%)$ choices toward the risky option. Of

2 note, the bias term in the explicit zero condition did not differ from 0 (mean $=0.02$,

$395 \% \mathrm{HDI}=[-0.06,0.10])$. In other words, in the explicit-zero condition, participants

4 may not have a notable prior preference for either option, and it was merely the value

5 difference that determined the choice of behavior. These results were in accordance

6 with our aforementioned behavioral results, suggesting that the explicit presentation

7 of zero outcomes in risky choices may shift the preference away from certain options

8 by highlighting the value difference between risky options and certain options.

9 Next, we designed Study 2 to thoroughly test the hidden-zero effect in a well-

10 controlled experiment, by using eye-tracking in combination with computational

11 modeling.

12

\section{Study 2}

The aim of Study 2 was to investigate the cognitive mechanisms of attention bias in the hidden-zero effect by incorporating process data into computational modeling. We used a risky decision paradigm similar to Study 1 while eye-movement was recorded.

\subsection{Method}

\subsubsection{Participants}

The initial sample comprised 60 undergraduate and postgraduate students $\left(\right.$ female $\left.=23, M_{\text {age }}=24.31, S D_{\text {age }}=1.89\right)$ from the research participant pool of the Institute of Psychology, Chinese Academy of Sciences. All the participants had a normal or corrected-to-normal vision and signed consent forms prior to the study. One participant was excluded from the analyses because of an incomplete eye-movement 


\section{HIDDEN-ZERO EFFECT IN RISKY DECISION-MAKING}

recording, thus, the final sample was 59 participants for the remaining analyses. Each participant was paid CNY 50 in cash for participation. To incentivize participants, they were endowed an additional amount as per their actual choices in one randomly selected trial.

\subsubsection{Apparatus}

Eye movements were monitored with an EyeLink 1000 Eye-tracker (SR Research, Canada), with the eye position sampled at $1000 \mathrm{~Hz}$. Displays were presented on a 19-inch CRT monitor running at a refresh rate of $75 \mathrm{~Hz}$ with a resolution of $1024 \times 768$ pixels. To minimize head motion, a chin rest located $60 \mathrm{~cm}$ away from the monitor was used. Viewed from this distance, the screen subtended a visual angle of $28^{\circ}$ horizontally and $21^{\circ}$ vertically. The eye-tracker was calibrated using a 9-point calibration procedure. A drift correction (operated by the experimenter) was performed before each trial. Participants' responses were recorded by logging button presses on the keyboard.

\subsubsection{Material and procedure}

We used a modified between-subject design and materials as in Study 1.

Participants were randomly assigned to either the hidden-zero $(n=30)$ or the explicitzero $(n=29)$ conditions. On the principle of parsimony, the experimental trials only remained the pairs of options containing relatively large or small probabilities (low risk, $5 \%-20 \%$, or high risk, $80 \%-95 \%$ ) from the risky options in Study 1 . To assure the display equivalence to the option pairs of the explicit zero condition, we used an "X" sign as placeholders for the zero outcomes (of the certain options and risky options) in the hidden-zero condition (e.g., X\% for CNY X, Fig. 3).

In each condition, the decision task comprised four 24-trial (1/3 were fillers) blocks, with the presentation mode counterbalanced across the blocks. That is, across different blocks, the zero outcomes or " $\mathrm{X}$ " signs of the risky and certain options were either presented on the left or right, constituting four different presentation modes 
HIDDEN-ZERO EFFECT IN RISKY DECISION-MAKING

1 (Fig. 3). The experimental trials were approximately or exactly equal across different

2 blocks. In addition, to ensure that participants could not perceive or identify more than

3 one attribute at once without making an eye movement (Rayner, 2009), any two of the

4 eight attributes ( 4 payoffs +4 probabilities) in a single stimulus lay in the periphery

5 (out of the central $5^{\circ}$ of vision). Therefore, each participant completed 116 trials, and

6 the order of blocks was counterbalanced across trials with a Latin square design.

7 In each block, five practice trials were first presented before testing to familiarize

8 participants with the task presentation. During the test session, the participants first

9 fixated on a cross at the center of the screen and then responded. Feedback and a

10 1,000-ms interval (with a blank screen) followed before the next trial. The order of the

11 trials in each block was randomized.

Different from Study 1, to control for the potential effect of dispositional risk

14 preference, participants completed the Risk Propensity Scale (RPS) (Meertens \&

15 Lion, 2008) after the decision task to measure their general risk-taking attitude.

16 Participants rated on a 9-point Likert scale, ranging from 1 (totally disagree) to 9

17 (totally agree), for the first six items (e.g., I do not take risks with my health). The last

18 item was rated on an inverse scale, ranging from 1 (risk avoider) to 9 (risk seeker).

19 Higher RPS scores indicated a higher risk-seeking attitude.

\section{$20 \quad$ 3.2. Results}

Eight non-overlapping, identically sized $(175 \times 159$ pixels $)$ rectangular regions

of interest were defined with each covering one choice attribute. Overall, 127 of 3,776

2454 trials $(1.4 \%)$ were discarded because of eye-tracking failures. Additionally, 81

$25(2.2 \%)$ trials were discarded because the decision time was shorter than $200 \mathrm{~ms}$ or

26 longer than three standard deviations from the mean. Thus, the final number of valid

27 trials was 3,641 . 


\section{HIDDEN-ZERO EFFECT IN RISKY DECISION-MAKING}

\subsubsection{Choice preference}

The same as in Study 1, we used binary logistic regression implemented in a generalized linear mixed model to predict participants' choices, with the same fixed effects (conditions and probabilities), and Subject ID and RPS scores $(M=3.29, S D=$ 1.04 , Cronbach $\alpha=.77$ ) as random effects. Given that some of the experimental trials were similar across four blocks, we put trial repeated times as the fixed effect covariance (Table A2). The effect of the condition was significant $(\beta=-.84, p=.01$, odds ratio $=.43,95 \% \mathrm{CI}=[.22-.83]$, Fig. 1$)$, indicating that individuals preference for a certain option decreased when the zero outcome was explicitly presented. Given the significant interaction of condition and probability $(\beta=-.82, p<.01$, odds ratio $=$ $2.26,95 \% \mathrm{CI}=[1.39-3.68])$, we divided trials further into high/low risky level groups. The similar generalized linear mixed model without the interaction effect indicated a marginal significant effect of condition only in high risky level group, $\beta_{h i g h}$ $=-.92, p_{\text {high }}=.09$, odds ratio $=.40,95 \% \mathrm{CI}=[.12-1.23] ; \beta_{\text {low }}=-.14, p_{\text {low }}=.97$. Taken together, the hidden-zero effect, as demonstrated in Study 1, was replicated, especially when individuals encountered higher risky level choices.

\subsubsection{Empirical attentional bias scores in attention allocation}

To measure the attention allocation pattern across different conditions, we computed the empirical attentional bias, using SB scores of option and outcomes levels based on the fixation data. The SB score represents a direct measure of overt attention to a specific element (Franco-Watkins et al., 2016). We calculated SB score based on dwell time ${ }^{1}$ to the area of interest (AOI) of certain and risky choice options at option and outcome levels (Figure 2). Specifically, at the option level, $\mathrm{SB}_{\text {option }}$

\footnotetext{
${ }^{1}$ In addition to the dwell time, we also calculated the SB score of fixation count. Given that the dwell time and fixation count have a high correlation $(r=.95 \sim .97, \mathrm{ps}<.001)$, and analyses using the fixation count data show the same effects (data not shown).
} 


\section{HIDDEN-ZERO EFFECT IN RISKY DECISION-MAKING}

1 represented the attentional bias to a certain or risky option; on the outcome level,

$2 \mathrm{SB}_{\text {zero_outcome }}$ and $\mathrm{SB}_{\text {non-zero_outcome }}$ represented the attentional bias to the zero (e.g.,

$390 \%$ CNY 100) or non-zero outcome (e.g., 10\% CNY 0) of the certain or risky option.

4 The formulation of SB scores was specified as follows:

$$
\mathrm{SB}_{\text {option }}=\frac{(\text { AOI of certain option })-(\text { AOI of risky option })}{\text { Total AOIS }}
$$

$$
\mathrm{SB}_{\text {zero_outcome }}=\frac{(\text { AOI of certain zero outcome })-(\text { AOI of risky zero outcome })}{\text { Total AOIs }}
$$

$$
\mathrm{SB}_{\text {non-zero_outcome }}=\frac{(\text { AOI of certain non zero outcome })-(\text { AOI of risky non zero outcome })}{\text { Total AOIs }}
$$

The SB scores ranged between -1 and +1 . An SB score of +1 indicated an extreme bias to the certain options (or outcomes), whereas -1 indicated an extreme bias to risky options (or outcomes). An SB score of 0 indicated no specific bias to either certain or risky options (or outcomes). A linear mixed regression model with the same fixed and random effects as that for choices was implemented to predict participants' SB scores (see Table A3).

Option level. The effect of condition and probability reached significance $\left(\beta_{\text {condition }}=-.18, p_{\text {condition }}<.001\right.$, odds ratio $=.84,95 \% \mathrm{CI}=[.79-.89] ; \beta_{\text {probability }}=$ - .09, probability $=.02$, odds ratio $=.91,95 \% \mathrm{CI}=[.85-.98])$, indicating that participants had congruously allocated more attention to certain options when they were blinded to zero outcomes and when the probability of risky choice was low. In addition, participants paid more attention to risky options when zero outcomes were experimentally explicit and when the probability of a risky choice was high. Their interaction was significant $(\beta=.09, p<.001$, odds ratio $=1.09,95 \% \mathrm{CI}=[1.04-$

1.15]). The effect of condition was significant for risky level $\left(\beta_{h i g h}=-.17\right.$, $p_{\text {high }}<.001$, 
HIDDEN-ZERO EFFECT IN RISKY DECISION-MAKING

1 odds ratio $=.85,95 \% \mathrm{CI}=[.78-.90] ; \beta_{\text {low }}=-.10$, $p_{\text {low }}=.004$, odds ratio $=.91,95 \%$

$2 \mathrm{CI}=[.85-.97])$, indicating that participants shifted their attention from a certain to

3 risky option when zero outcome was presented. Moreover, comparing the mean SB

4 score against 0 (two-tailed one sample $t$-test) showed that in the explicit-zero

5 condition, participants' SB scores were smaller than 0 (higher risky levels, $t(28)=-$

$65.43, p<.001$; lower risky levels, $t(28)=-3.20, p=.003)$, indicating an attentional

7 bias in favor of the risky option; contrarily, in the hidden-zero condition, participants'

8 SB scores were larger than 0 but only at the higher risky levels, $t(29)=2.64, p=.01$

9 (in lower risky levels $(t(29)=1.71, p=.10)$, indicating an attentional bias in favor of a

10 certain option.

11 Outcome level. For zero outcomes, the effect of condition, probability, and

12 interaction were significant $\left(\beta_{\text {condition }}=-.10\right.$, $p_{\text {condition }}<.001$, odds ratio $=.91,95 \% \mathrm{CI}$

$13=[.89-.92] ; \beta_{\text {probability }}=-.05, p_{\text {probability }}=.005$, odds ratio $=.95,95 \% \mathrm{CI}=[.92$

$14-.98] ; \beta_{\text {interaction }}=.05, p_{\text {interaction }}<.001$, odds ratio $\left.=1.06,95 \% \mathrm{CI}=[1.03-1.08]\right)$. The

15 effect of condition was significant at the risky level $\left(\beta_{\text {high }}=-.09, p_{h i g h}<.001\right.$; odds

16 ratio $=.91,95 \% \mathrm{CI}=[.90-.93] ; \beta_{\text {low }}=-.05, p_{\text {low }}<.001 ;$ odds ratio $=.95,95 \% \mathrm{CI}=$

17 [.94 - .97]), indicating that participants paid more attention to the zero outcomes of a

18 risky option when a zero outcome was presented. Results of two-tailed one sample $t$ -

19 test showed that SB scores of explicit-zero condition were significantly smaller than 0

20 (higher risky levels, $t(28)=-12.46$; lower risky levels, $t(28)=-11.16, p s<.001$ ).

21 Altogether, the results indicated that in an explicit-zero condition, participants paid

22 more attention to risky zero outcomes.

23 Regarding the non-zero outcomes, the effect of condition and the interaction

24 were significant or marginally significant $\left(\beta_{\text {condition }}=-.08, p_{\text {condition }}=.01\right.$, odds ratio

$25=.92,95 \% \mathrm{CI}=[.87-.98] ; \beta_{\text {interaction }}=.03, p_{\text {interaction }}=.09$, odds ratio $=1.04,95 \% \mathrm{CI}$

$26=[.99-1.08])$. The effect of condition was significant at the higher risky level $\left(\beta_{\text {high }}\right.$

$27=-.08, p_{\text {high }}=.01 ;$ odds ratio $=.93,95 \% \mathrm{CI}=[.88-.98] ; \beta_{\text {low }}=.05$, $p_{\text {low }}=.10$, odds 


\section{HIDDEN-ZERO EFFECT IN RISKY DECISION-MAKING}

ratio $=.95,95 \% \mathrm{CI}=[.90-1.00])$, indicating that participants paid more attention to the non-zero outcomes of a risky option at a higher risky level when presenting zero outcomes. SB scores in the hidden-zero condition were significantly larger than 0 (two-tailed one sample $t$-test, higher risky levels, $t(29)=3.15, p=.004$, lower risky levels, $t(29)=2.54, p=.02)$. SB scores in the explicit-zero condition did not differ from 0 (higher risky levels, $t(28)=-.17, p=.87$; lower risky levels, $t(28)=1.52, p=.10$ ). Altogether, these results indicated that when zero outcomes were hidden, individuals allocated more attention to non-zero outcomes of certain options than those of risky options. However, explicitly presenting zero outcomes eliminated such attentional bias.

(1)

(1)

\subsubsection{Computational Modeling}

\subsubsection{1 candidate models}

4 To compare the models with or without eye-movement data, we first fit the same models that were described in Study 1 to participants' choice data in the hidden-zero (M11 and M12) and explicit-zero (M21 and M22) conditions, respectively. Given the unique features of the eye-movement data, where a trial-wise trace process was precisely measured, we incorporated the three aforementioned SB scores into the computational models for both conditions to examine whether attention played a mechanistic role in the hidden-zero effect. Hence, we tested six models each for the 1 hidden-zero condition (M11-M16) and the explicit-zero condition (M21-M26). Both 2 sets of models (for both conditions) started with the base model (M11/M21), that is, 3 the RA model or the RA- $p \lambda$ model, respectively. M12/M22 included a simple 4 descriptive attentional bias (denoted as $\beta_{0}$, as in Study 1). On top of that, M13-M16 5 (and M23-M26) incorporated different SB scores derived from eye-movement data, 6 namely, $\mathrm{SB}_{\text {option, }} \mathrm{SB}_{\text {zero_outcome, }} \mathrm{SB}_{\text {non-zero_outcome, }}$ and $\mathrm{SB}_{\text {zero_outcome }}+\mathrm{SB}_{\text {non-zero_outcome }}$ 7 (denoted as $\mathrm{SB}_{\text {zero_outcome_none_zero_outcome }}$ thereafter), respectively. Given that we could 


\section{HIDDEN-ZERO EFFECT IN RISKY DECISION-MAKING}

formally test the effect size and contributions of each SB score, the newly constructed models thus provided stronger process implication than the pure attentional bias models (aRA and aRA-p $\lambda$, subsection 2.1.3) alone. By selecting the winning model, we could also examine which SB score contributed to the altered cognitive processes; hence, the consequent choice preference.

Model estimation and model comparison procedures remained the same as in Study 1 (subsection 2.2.2) except that we reconstructed the attentional bias by including SB scores in the Softmax function (Eq. 4). Because the SB scores entailed specific attentional features, the newly constructed bias term suggested an empirical attentional bias (relative to the descriptive attentional bias in Study 1). Notably, regarding the principle of parsimony, we included only SB scores that had significant results in the previous analysis in the subsequent computational modeling analysis (Table A3).

\subsubsection{Model estimate and model comparison results}

Similar to Study 1, model comparison results revealed that introducing an attentional bias (M12-M16/M22-M26, relative to M11/M21) provided a notable improvement in the model estimate in the hidden-zero and the explicit-zero conditions. Strikingly, accommodating eye-movement attentional SB (i.e., SB scores; M13-M16/M23-M26) further enhanced model performance in both conditions. In particular, the model that integrated two SB scores at the outcome levels (i.e.,

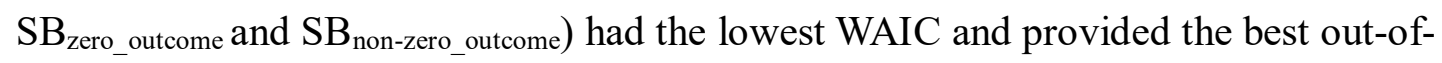
sample predictive accuracy (M16/M26; Fig. 5A/D) for both conditions.

To select the final winning model, we then compared the models based on the psychological features of those SB scores, given that not all differences between models incorporating corresponding SB scores (M13-M16 / M23-M26) on the WAIC scale were greater than the conventional decision margin $(\Delta \mathrm{WAIC}=10$; Kass \& Raftery, 1995; Burnham \& Anderson; 2004). First, compared with the $\mathrm{SB}_{\text {option, the two }}$ 
HIDDEN-ZERO EFFECT IN RISKY DECISION-MAKING

1 SB $\mathrm{B}_{\text {outcome }}$ scores $\left(\mathrm{SB}_{\text {zero_outcome }}\right.$ and $\left.\mathrm{SB}_{\text {non_zero_outcome }}\right)$ provided a more detailed and

2 focused attentional bias configuration and retained smaller AOI; additionally, they

3 (i.e., zero and non-zero) shared a common eye-movement feature with the $\mathrm{SB}_{\text {option. }}$.

4 Consistent with these features, our model comparison results also showed that

5 simultaneously incorporating $\mathrm{SB}_{\text {zero_outcome }}$ and $\mathrm{SB}_{\text {non_zero_outcome was superior regarding }}$

6 model performance $\left(\triangle \mathrm{WAIC}<0\right.$, with reference to $\left.\mathrm{SB}_{\text {option }}\right)$, whereas merely

7 incorporating either $\mathrm{SB}_{\text {zero_outcome }}$ or $\mathrm{SB}_{\text {non_zero_outcome }}$ did not provide better model

8 performance (both $\triangle$ WAIC $>0$, with reference to $\mathrm{SB}_{\text {option }}$ ). Considering that both

$9 \mathrm{SB}_{\text {outcome }}$ scores provided independent contributions to the model's performance, the

10 effect size of $\mathrm{SB}_{\text {zero_outcome }}+\mathrm{SB}_{\text {non-zero_outcome }}$ did not show any significant correlations

11 (in the hidden-zero condition, $r=-0.020, p>0.1$; in the explicit-zero condition, $r=$ -

$120.049, p>0.1)$, we concluded the aRA(- $p \lambda)+\mathrm{SB}_{\text {zero_outcome }}+\mathrm{SB}_{\text {non-zero_outcome }}$ model as

13 the winning model.

14 Finally, following the same procedure in Study 1, we tested whether the winning

15 model was able to recapitulate individuals' behavioral pattern. By comparing posterior

16 model predictions with the actual choices, we demonstrated that models integrating

$17 \mathrm{SB}_{\text {zero_outcome }}$ and $\mathrm{SB}_{\text {non_zero_outcome }}$ (in hidden-zero condition, M16; in explicit-zero

18 condition, M26) indeed well captured participants' choice preference (see Fig. 5C/F).

19 These results indicated that, in both explicit- and hidden-zero conditions, participants'

20 attention was largely biased toward the non-zero outcomes. Intriguingly and in

21 concurrence with our Study 1 findings, in the explicit-zero condition, the additional

22 bias toward the certain zero outcome was diminished compared with the hidden-zero

23 condition (the corresponding $\beta$ values were negative; hence, the smaller the $\beta$, the

24 more likely an individual was to choose certain options; independent samples t-test,

$25 t(45)=44.48, P<0.001)$, suggesting an enhanced bias in favor of the risky option

26 when zero outcomes were presented. In addition, the choice preference in the explicit-

27 zero condition was also modulated by participants' aversion $(\lambda)$ to the probability of 
HIDDEN-ZERO EFFECT IN RISKY DECISION-MAKING

1 obtaining a void outcome, which was not the case in the hidden-zero condition (Fig.

$25 \mathrm{E})$.

\section{General Discussion}

We investigated the hidden-zero effect in risky decision-making and developed an attentional risk aversion (aRA) model to examine its process mechanism by combining hierarchical Bayesian modeling and eye-tracking. In Study 1, we verified the presence of the hidden-zero effect and observed that when presenting the zero outcomes for a risky choice, individuals' choice preference toward certain options could be eliminated, namely, the certainty effect, and preference could be shifted by a descriptive attentional bias toward certain options. In Study 2, with the effect of individual differences of risk avoidance traits controlled, we observed that when explicitly presenting zero outcomes led choice preference and attention toward risky options. Computationally, while evolving empirical attentional bias (i.e., SB scores) to zero as well as non-zero outcomes in the modeling, the model predicted the choice preference more precisely than other candidate models regardless of hidden or explicit present zero outcomes. These results suggest that an attentional bias toward outcomes alters the attentional allocation to risky and certainty options in risky decisionmaking, and presenting the zero outcomes may lead to an unmixed effect of probability aversion in risky choices. 


\section{HIDDEN-ZERO EFFECT IN RISKY DECISION-MAKING}

\subsection{Hidden-zero effect of risky choice}

Our results indicated that the zero component has indispensable consequences on individuals' risky decision-making preference and process. Although the value of zero outcomes is an absolute null event for a rational decision-maker, it entails a decreased choice proportion of a certain option by reducing the bias toward it and induces increased attention to the zero component of a risky option. This may challenge the prediction of EU-based risky choice models.

This finding identified the existence of the hidden-zero effect in risky choice, which is in consensus with previous results of zero outcomes in risky and intertemporal choice fields. Our results were consistent with Zhang and Slovic (2019), who reported a zero effect in the loss frame, that is, presenting a zero outcome pushes individuals to choose based on the EV of options; except that in our study, the zero effect is in the gain frame. Our results were consistent with those in intertemporal choice, given that the SS option and LL option in intertemporal choice correspond to a certain option and a risky option in risky choice, respectively. Presenting zero outcomes decreases participates' willingness to choose impatient choices (Magen et al., 2008; Read et al., 2016). However, our results slightly differ from the findings of Kühberger and Tanner (2010), that is, eliminating the zero component increased the choice proportion of a risky option. This difference may have been caused by the range of risky levels. In Kühberger and Tanner (2010), their risky levels were limited to $1 / 3$; in our study, we systematically set a relatively larger range of risky levels, from $5 \%$ to $95 \%$.

We further identified the possible boundary conditions of the hidden-zero effect in risky choices, that is, the size of the hidden-zero effect might be shaped together by the risky level and dispositional risk preference. One major difference between Study 1 and Study 2 is that the hidden-zero effect is relatively stronger at the higher risky level than that at the lower risky level in Study 2. Given that the risky level was quite 


\section{HIDDEN-ZERO EFFECT IN RISKY DECISION-MAKING}

1 close in the two studies, one possible reason is the participants in Study 2 were

2 relatively risk avoidant compared with those in Study 1. The participants in Study 2

3 choose more risky avoidance choices (between 13\% and 37\% across all conditions)

4 than in Study 1, and their PRS scores were lower than 5 points (out of 9 points, $M=$

$53.29, t=-12.67, p<.001)$. Moreover, in Study 2, participants were paid based on their

6 actual choices during the eye-tracking experiment, which may have led to a higher

7 tendency for risk avoidance. Therefore, we could infer that the hidden-zero effect is

8 more likely to be observed at a higher risky level and within populations that are more

9 risk avoidant. This inference might indicate that it is a unidirectional effect, that is,

10 decreasing the level of risk avoidance, not increasing risk approaching.

11 In addition, the core of our cognitive models is derived from PT. We

12 demonstrated adaptiveness and generalizability of the PT model family and that the

13 PT model family can account for a large range of risky decision-making paradigms.

14 Notably, although these finding may challenge EU-based models, we did not

15 invalidate PT in general, but rather, indicated that these models have an applicable

16 boundary condition, that is, they are not applicable to conditions when zero outcomes

17 are explicitly presented.

18 In summary, our results implied that the zero component should not be neglected

19 in research on risky decision-making. Despite the numerical value of zero outcomes

20 being void, it nonetheless affects choice preference by changing the information

21 processing of decision-making.

\subsection{Mechanism of the hidden-zero effect of risky choice}

In this study, we developed an attentional RA model and provided evidence that

24 a pronounced attentional bias (both descriptively and empirically) toward certain

25 options in the hidden-zero condition and explicitly presenting zero outcomes might

26 eliminate this bias to certain options in risky decision-making. These results suggested

27 the attentional bias may play a central role in the mechanism of the hidden-zero effect 
HIDDEN-ZERO EFFECT IN RISKY DECISION-MAKING

in risky choice.

Specifically, our results indicated that attention could moderate the information

3 processing of the option attributes. In Study 2, explicitly presenting zero outcomes

4 drew more attention to a risky option and then entailed a decreased preference for

5 certain options. This mechanism was consistent with the new line of research that has

6 highlighted the role of attentional bias in decision-making. The closest example is

7 from Read and colleagues (2016), who explained the hidden-zero effect in

8 intertemporal choice such that individuals have an attentional bias to the zero outcome

9 of SS option and may alter choice preference. Kreiner and his colleagues (2018) also

10 observed that shifting the attention of the message recipient to the complementary

11 frame would reduce or eliminate attribute-framing bias, that is, a tendency to evaluate

12 objects framed positively (75\% success) more than objects framed negatively $(25 \%$

13 failure). Pachur and his colleagues (Pachur, Schulte-Mecklenbeck, Murphy \&

14 Hertwig, 2018) connected measures of attention process to the CPT and observed that

15 attention allocation causes choice regularities that can be captured by CPT. Compared

16 to these studies that mainly focused on an interpretational approach, our results, by

17 using computational modeling in both studies, consistently demonstrated the

18 quantitative contribution of attention to predicting behavioral regularity. Specifically,

19 at the behavioral level, model evidence assessed by WAIC suggested quantitative

20 improvement when including the empirical attentional bias. After including eye-

21 tracking attention in the modeling (Study 2 only), this contribution of attention was

22 manifested by a sizable decrease in the WAIC scale. Thus, our results indicated that

23 attention played a key role in the hidden-zero effect in risky decision-making, which

24 would be reflected by the increased model performance quantitatively and

25 qualitatively.

26 4.3. Model development combining behavioral and eye-movement data

27 Our study showed enhanced predictive power in predicting choice preference 


\section{HIDDEN-ZERO EFFECT IN RISKY DECISION-MAKING}

and provided a novel approach to model development by accommodating important process evidence (i.e., SB scores derived from eye-movement data) in our cognitive models. Our model has several advantages at the methodological level.

First, we incorporated process and behavioral data in our model fitting framework. This attempt provided a potentially richer and more powerful path compared with traditional behavioral-based models. The literature on cognitive modeling had mainly fit to behavioral data (Chávez, Villalobos, Baroja, \& Bouzas, 2017; Dai, Pleskac, \& Pachur, 2018; Wulff \& van den Bos, 2017) or used single/multiple eye-movement features to predict behavior (Krajbich, Armel, \& Rangel, 2010). These practices could explain the behavioral effect at large but did not consider the combined power across modalities, for instance, when eye movement was recorded alongside behaviors. Many researchers have addressed that decision models should make detailed predictions about cognitive processes, rather than merely predicting decisional outcomes, and provide a priori predictions rather than post hoc descriptions (Marewski \& Bröder, 2018). However, because of the prevailing outcome-based model-testing strategy, most decision models (i.e. PT theory) based on preference have not presented explicit hypotheses on their underlying cognitive process. Our endeavor of considering choice preference and attentional process in model estimation might provide a method to avoid such an impasse: either be confirmed or falsified simply by using several paradoxical cognitive processes as evidence, such as fixation numbers or durations. Moreover, in comparison with the data-driven strategy in a previous model fitting, accommodating eye-movement data in the model fitting help us distinguish candidate models based on the psychological implication of the specific eye-movement index, as is the case in Study 2 (section 3).

5 That is, when model parsimonies (e.g., AIC, WAIC) reveal comparable and indecisive results, researchers should select models by considering each model's psychological implications (Lewandowsky \& Farrell, 2010). When model comparison fails to favor 


\section{HIDDEN-ZERO EFFECT IN RISKY DECISION-MAKING}

one model over another, considering whose psychological implication is theoretically and conceptually more plausible and may open a new perspective in model evaluation.

\subsection{Contributions and implications}

Our research set a relatively large range of risky levels to systematically examine the hidden-zero effect in risky choice and proposed attention as its underlying cognitive mechanism. Theoretically, our aRA model offers a more integrative computation account to understand the role of hidden-zero outcome in risky decisionmaking. These findings compensate for the lack of zero components in classic decision models that take zero outcomes as irrational components. Additionally, we extend the boundary of fuzzy-trace theory beyond the framing effect in a larger range of risky levels.

Our findings also supply a potential means to manipulate an individual's preferences in risky settings. Given that the attentional bias may shift an individual's choice preference in risky decision-making depending on whether zero outcomes are presented, the hidden-zero effect could be used as a cheap but effective behavior nudge to encourage high-risk decisions. For example, delivering potential void outcomes may promote risk-taking, for instance, when opening new markets and adopting new production methods to improve enterprise innovation decisions.

Computationally, our attentional RA model accommodates process features to disentangle the potential role of attentional bias in the computational processes underlying the hidden-zero effect. Our process models make predictions based on observed information (e.g., choice and outcome) and the information of the latent underlying processes (e.g., attention). These advantages make process models superior for explaining the underlying behavioral effects and quantitatively evaluating the candidate models based on their psychological implications. This model fitting framework may provide new insights into future decision-making modeling. 
HIDDEN-ZERO EFFECT IN RISKY DECISION-MAKING

\section{$1 \quad 4.5$ Limitations}

\section{Conclusion}

In summary, this study verified the existence of the hidden-zero effect in risky decision-making. Using hierarchical Bayesian modeling, we proposed an attentional risk aversion model combining behavioral and trace processes to reveal the underlying cognitive computational mechanism of this effect. Our results reveal that an attentional bias in favor of a risky option when zero outcomes are presented may play a decisive role as the potential mechanism of zero effect. This effect is perhaps applicable to encouraging risk-taking behaviors when making political and economic decisions. 
HIDDEN-ZERO EFFECT IN RISKY DECISION-MAKING

6 German Research Foundation (Deutsche Forschungsgemeinschaft, Research Training

7 Group: CINACS, GRK 1247), and the Research Promotion Fund (FFM) for young

8 scientists of the University Medical Center Hamburg-Eppendorf.

11 Psychology, Chinese Academy of Sciences and Jia-Ying Xiao from Beijing Normal

12 University for their contribution to the literature reviewing. 


\section{References}

Ahn, W.-Y., Haines, N., \& Zhang, L. (2017). Revealing neuro-computational mechanisms of reinforcement learning and decision-making with the hBayesDM package. bioRxiv, 1, 24-57. doi: 10.1162/cpsy_a_00002

Ahn, W.-Y., Krawitz, A., Kim, W., Busemeyer, J. R., \& Brown, J. W. (2011). A model-based fMRI analysis with hierarchical Bayesian parameter estimation. Journal of Neuroscience, Psychology, and Economics, 4, 95-110. doi:10.1037/a0020684

Brandstätter, E., \& Körner, C. (2014). Attention in risky choice. Acta Psychologica, 152, 166-176. doi: 10.1016/j.actpsy.2014.08.008

Burnham, K. P., \& Anderson, D. R. (2004). Multimodel inference: Understanding AIC and BIC in model selection. Sociological Methods \& Research, 33, 261-304. doi: $10.1177 / 0049124104268644$

Chávez, M. E., Villalobos, E., Baroja, J. L., \& Bouzas, A. (2017). Hierarchical Bayesian modeling of the English premier league. Judgment and Decision Making, 12, 1928. doi: $10.1177 / 0956797616664342$

Dai, J., Pleskac, T. J., \& Pachur, T. (2018). Dynamic cognitive models of intertemporal choice. Cognitive Psychology, 104, 29-56. doi: 10.1016/j.cogpsych.2018.03.001

Fisher, G., \& Rangel, A. (2013, Oct.). Intertemporal discount rates are mediated by relative attention. Paper presented at the 34th Society for Judgment and Decision Making Annual Conference, Toronto, Canada.

Franco-Watkins, A. M., Mattson, R. E., \& Jackson, M. D. (2016). Now or later? Attentional processing and intertemporal choice. Journal of Behavioral Decision Making, 29, 206-217. doi: 10.1002/bdm.1895

Gelman, A., Carlin, J. B., Stern, H. S., Dunson, D. B., Vehtari, A., \& Rubin, D. B. (2014). Bayesian data analysis (3rd ed.). New York, NY: CRC Press. 
HIDDEN-ZERO EFFECT IN RISKY DECISION-MAKING

1 Gelman, A., \& Rubin, D. B. (1992). Inference from iterative simulation using multiple sequences. Statistical Science, 7, 457-472. doi: 10.1214/ss/1177011136

Kahneman, D., \& Tversky, A. (1979). Prospect theory: An analysis of decision under risk. Econometrica: Journal of the Econometric Society, 47, 263-291. doi: 10.1111/j.1536-7150.2011.00774.x

Kass, R. E., \& Raftery, A. E. (1995). Bayes factors. Journal of The American Statistical Association, 90, 773-795.

Krajbich, I., Armel, C., \& Rangel, A. (2010). Visual fixations and the computation and comparison of value in simple choice. Nature Neuroscience, 13, 1292-1298. doi: $10.1038 / \mathrm{nn} .2635$

Krajbich, I., Lu, D., Camerer, C., \& Rangel, A. (2012). The attentional drift-diffusion model extends to simple purchasing decisions. Frontiers in Psychology, 3. doi: 10.3389/fpsyg.2012.00193

Krajbich, I., \& Rangel, A. (2011). Multialternative drift-diffusion model predicts the relationship between visual fixations and choice in value-based decisions. Proceedings of the National Academy of Sciences, 108, 13852-7. doi: 10.1073/pnas.1101328108

Kreiner, H., \& Gamliel, E. (2018). The role of attention in attribute framing. Journal of Behavioral Decision Making, 31, 392-401. doi: 10.1002/bdm.2067

Kühberger, A., \& Tanner, C. (2010). A test of prospect theory and fuzzy-trace theory in risky choice framing. Journal of Behavioral Decision Making, 23, 314-329. doi: 10.1002/bdm.656

Lewandowsky, S., \& Farrell, S. (2010). Computational modeling in cognition: Principles and practice. New York, NY: Sage.

Magen, E., Dweck, C. S., \& Gross, J. J. (2008). The hidden-zero effect: Representing a single choice as an extended sequence reduces impulsive choice. Psychological Science, 19, 648-649. doi: 10.1111/j.1467-9280.2008.02137.x 
HIDDEN-ZERO EFFECT IN RISKY DECISION-MAKING

Marewski, J. N., \& Bröder, A. (2018). Some metatheoretical reflections on adaptive decision making and the strategy selection problem. Journal of Behavioral Decision Making, 198, 181-198. doi: 10.1002/bdm.2075

Meertens, R. M., \& Lion, R. (2008). Measuring an individual's tendency to take risks: The risk propensity scale. Journal of Applied Social Psychology, 38, 1506-1520. doi: 10.1111/j.1559-1816.2008.00357.x

Pachur, T., Schulte-Mecklenbeck, M., Murphy, R. O., Hertwig, R. (2018). Prospect theory reflects selective allocation of attention. Journal of Experimental Psychology: General, 147(2), 147-169. doi: 10.1037/xge0000406

Palmeira, M. M. (2011). The zero-comparison effect. Journal of Consumer Research, 38, 16-26. doi: 10.1086/657998

Pascal, B. (1670). Pensées (W. F. Trotter, Trans.). Retrieved October 25, 2012, from http://oregonstate.edu/instruct/phl302/ texts/pascal/pensees-contents.html.

Pärnamets, P., Johansson, P., Hall, L., Balkenius, C., Spivey, M. J., \& Richardson, D. C. (2015). Biasing moral decisions by exploiting the dynamics of eye gaze. Proceedings of the National Academy of Sciences, 112, 4170-4175. doi: 10.1073/pnas.1415250112

Radu, P. T., Yi, R., Bickel, W. K., Gross, J. J., \& McClure, S. M. (2011). A mechanism for reducing delay discounting by altering temporal attention. Journal of the Experimental Analysis of Behavior, 96, 363-385. doi: 10.1901/jeab.2011.96-363

Read, D., Olivola, C., \& Hardisty, D. (2016). The value of nothing: Asymmetric attention to opportunity costs drives intertemporal decision making. Management Science, 63, 4277-4297. doi: 10.1287/mnsc.2016.2547

Schulte-Mecklenbeck, M., \& Kühberger, A. (2014). Out of sight-out of mind? Information acquisition patterns in risky choice framing. Polish Psychological Bulletin, 45, 21-28. doi: 10.2478/ppb-2014-0004

Sokol-hessner, P., Camerer, C. F., \& Phelps, E. A. (2013). Emotion regulation reduces 
HIDDEN-ZERO EFFECT IN RISKY DECISION-MAKING

loss aversion and decreases amygdala responses to losses. Social Cognitive and Affective Neuroscience, 8, 341-350. doi: 10.1093/scan/nss002

Sokol-Hessner, P., Hsu, M., Curley, N. G., Delgado, M. R., Camerer, C. F., \& Phelps, E. a. (2009). Thinking like a trader selectively reduces individuals' loss aversion. Proceedings of the National Academy of Sciences, 106, 5035-5040. doi: 10.1073/pnas.0806761106

Sutton, R. S., \& Barto, A. G. (1998). Reinforcement learning: An introduction. MIT Press. doi: 10.1109/TNN.1998.712192

Tversky, A., \& Kahneman, D. (1992). Advances in prospect theory: Cumulative representation of uncertainty. Journal of Risk and Uncertainty, 5, 297-323. doi: 10.1007/BF00122574

Vehtari, A., Gelman, A., \& Gabry, J. (2017). Practical Bayesian model evaluation using leave-one-out cross-validation and WAIC. Statistics and Computing, 27, 14131432. doi: 10.1007/s11222-016-9696-4

von Neumann, J., \& Morgenstern, O. (1947). Theory of games and economic behavior. Princeton, NJ: Princeton University Press.

Zhang, Y., \& Slovic, P. (2019). Much ado about nothing: The zero effect in life-saving decisions. Journal of Behavioral Decision Making, 32, 30-37. doi: 10.1002/bdm.2089 


\section{Figure legend}

Figure 1. Choice proportion of a certain option in the different probabilities of each condition in Study 1 (top panel) and Study 2 (bottom panel).

Figure 2. Model evidence and parameter inference of computational models in Study 1.

Figure 3. The experimental trial and AOIs in the (A) explicit-zero and (B) hidden-zero conditions of Study 2. Solid boxes, the AOIs for option A; dotted boxes, the AOIs for option B; yellow boxes, the AOIs for non-zero outcome; red boxes, the AOI for zero outcome.

Figure 4. SB scores of different levels in the experimental conditions of Study 2 (A, option level; B, outcome level for non-zero outcomes; C, outcome level for zero outcomes).

Figure 5. Model evidence and parameter inference of computational models in Study 2. 
HIDDEN-ZERO EFFECT IN RISKY DECISION-MAKING
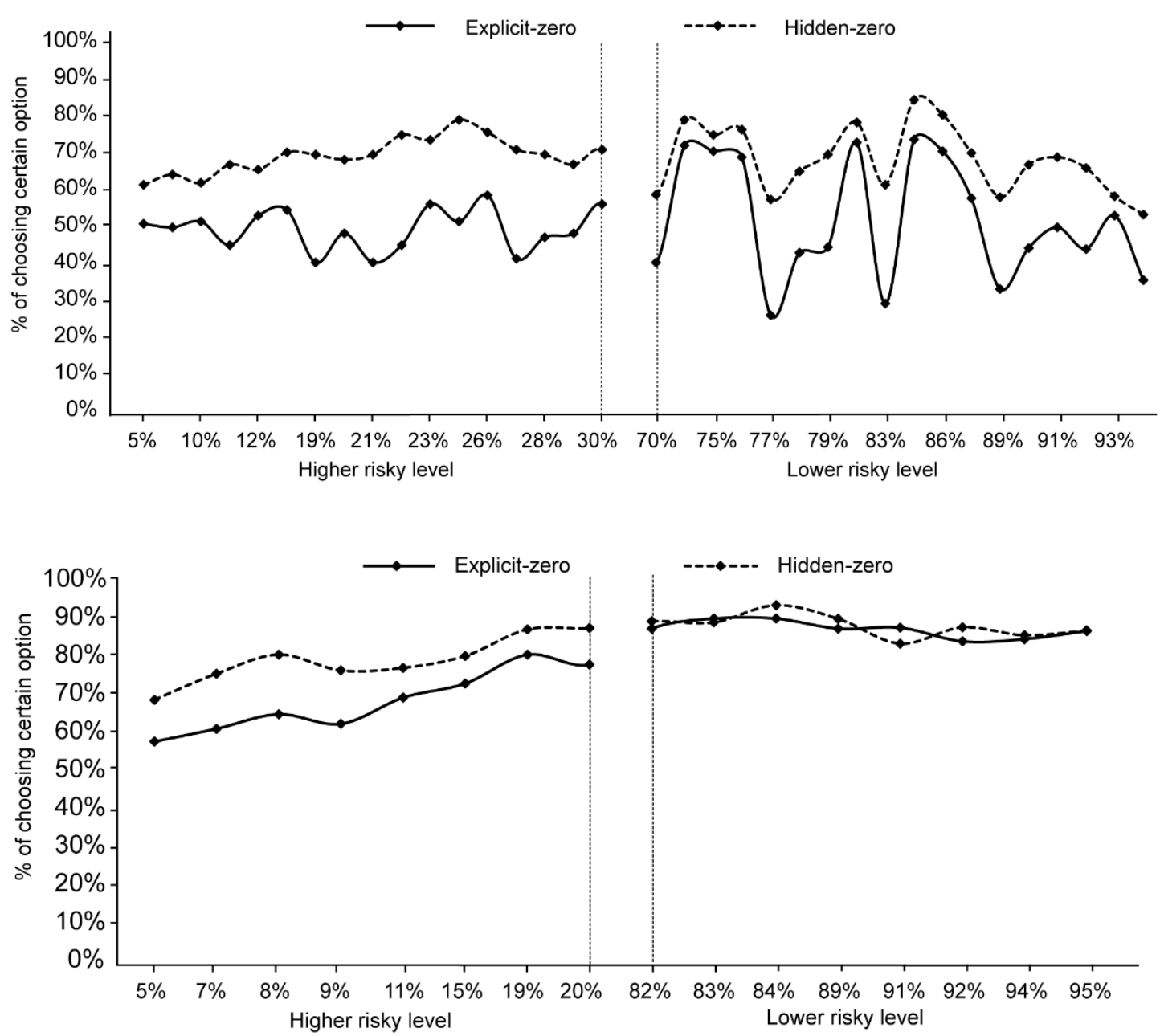

Figure 1. Choice proportion of a certain option in the different probabilities

4 of each condition in Study 1 (top panel) and Study 2 (bottom panel). 


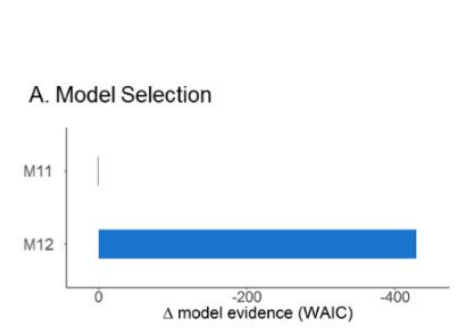

Hidden-zero condition

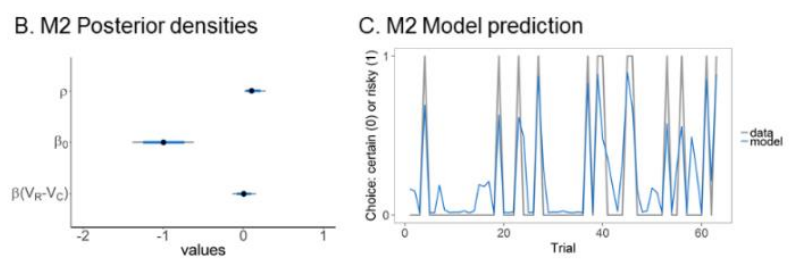

Explicit-zero condition
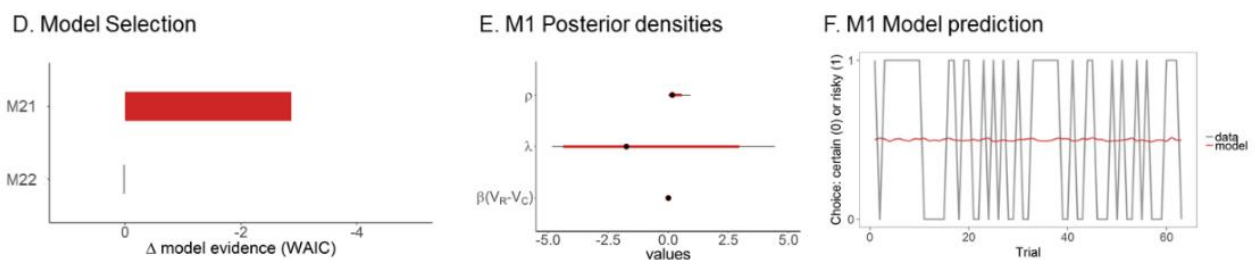

3 Study 1.

Figure 2. Model evidence and parameter inference of computational models in

Top panel: hidden-zero condition. (A) Model evidence (WAIC) with respect to M11 (RA model) favors M12 (shown in blue). Lower WAIC (i.e., more negative) indicates better out-of-sample predictive accuracy. (B) Posterior density of the winning model M12. Blue bars show the 95\% highest density interval (HDI). (C) Posterior model prediction of the winning model M12 is to verify whether the model (blue line) recapitulates the behavioral data (gray line) for an example participant. The modelgenerated prediction well matched the example participant's actual choices.

Bottom panel: explicit-zero condition. (D) Model evidence (WAIC) with respect to M22 (shown in red) favors M21. Although the difference in WAIC between M21 and M22 is relatively small, we adopt the "Occam's Razor" principle to choose the simpler model (M21). (E) Posterior density of the winning model M21. Red bars show the $95 \%$ highest density interval (HDI). (F) Posterior model prediction of the winning model M21 to verify whether the model (red line) recapitulates the behavioral data (gray line)

for an example participant. 
HIDDEN-ZERO EFFECT IN RISKY DECISION-MAKING

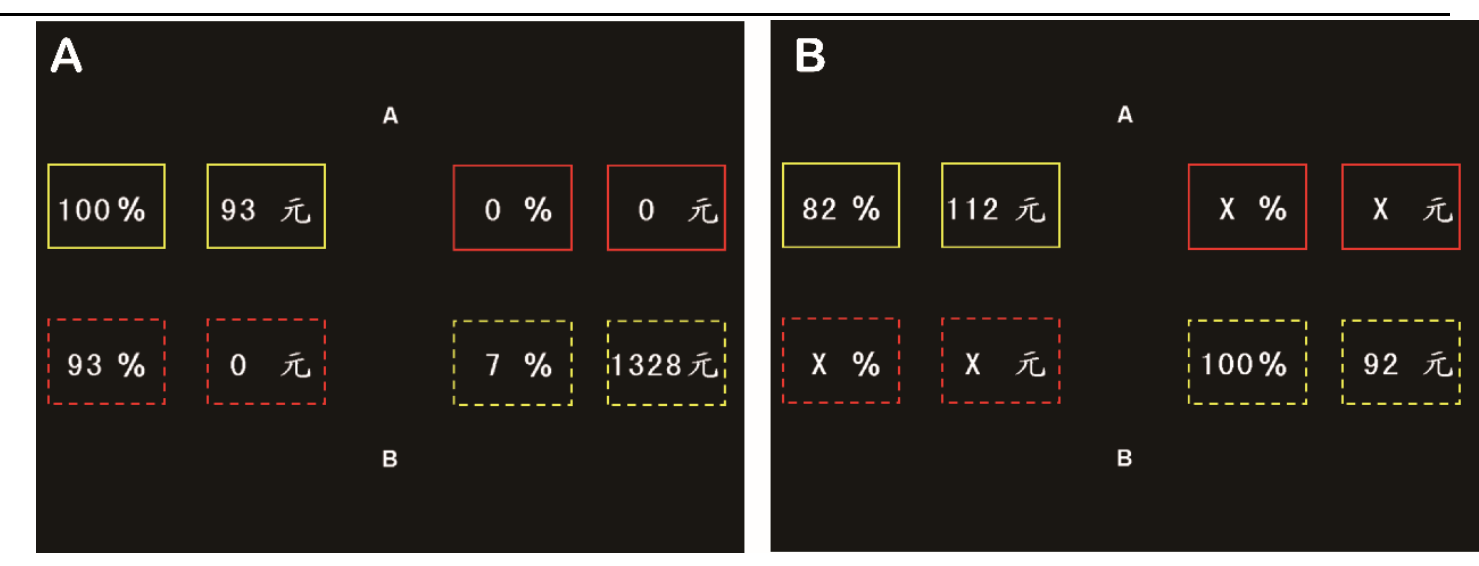

Figure 3. The experimental trial and AOIs in the (A) explicit-zero and (B)

3 hidden-zero conditions of Study 2. Solid boxes, the AOIs for option A; dotted

4 boxes, the AOIs for option B; yellow boxes, the AOIs for non-zero outcome; red

5 boxes, the AOI for zero outcome.

6 
HIDDEN-ZERO EFFECT IN RISKY DECISION-MAKING
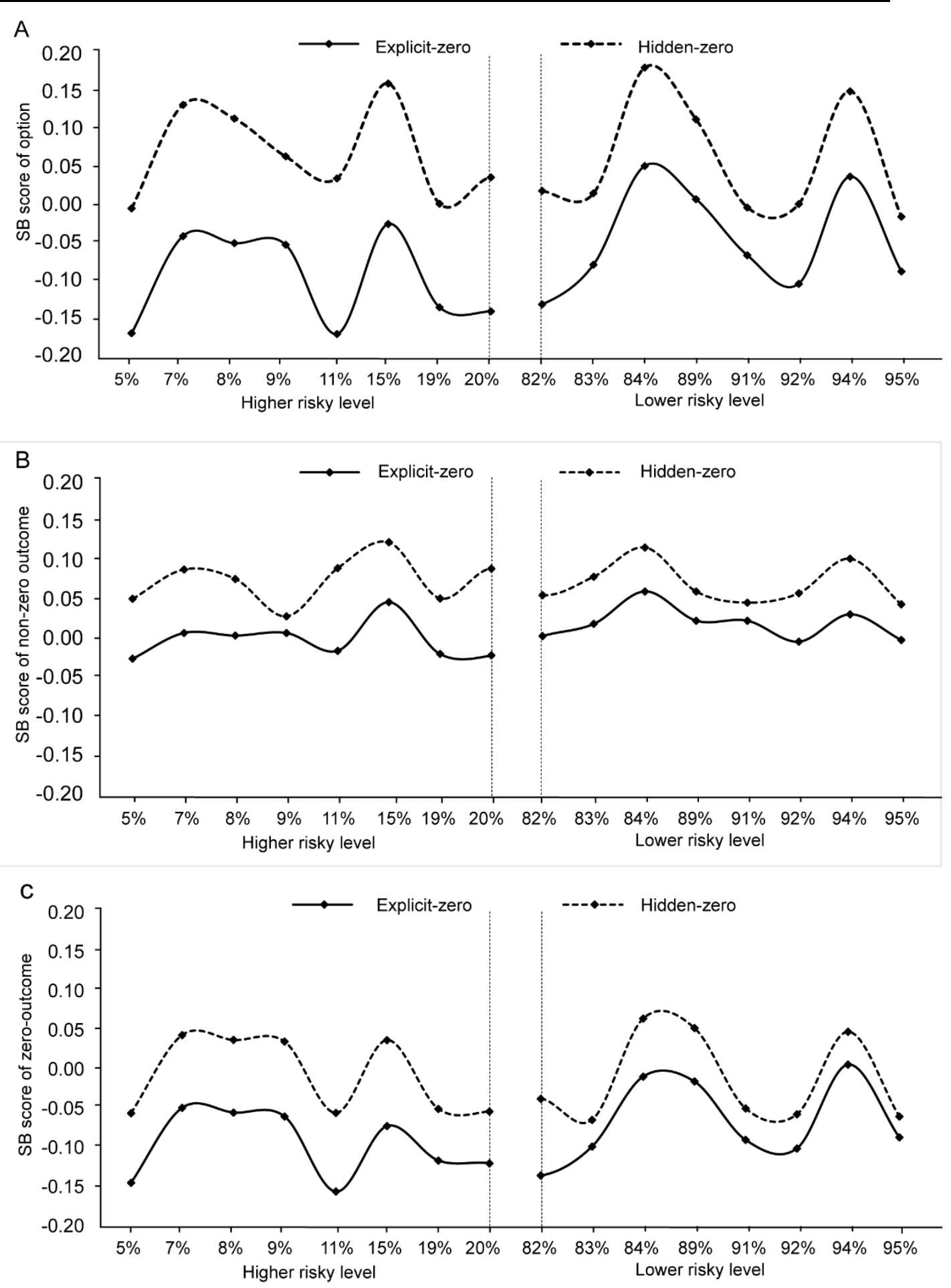

Figure 4. SB scores of different levels in the experimental conditions of Study 2 (A, option level; B, outcome level for non-zero outcomes; C, outcome level for zero outcomes). 


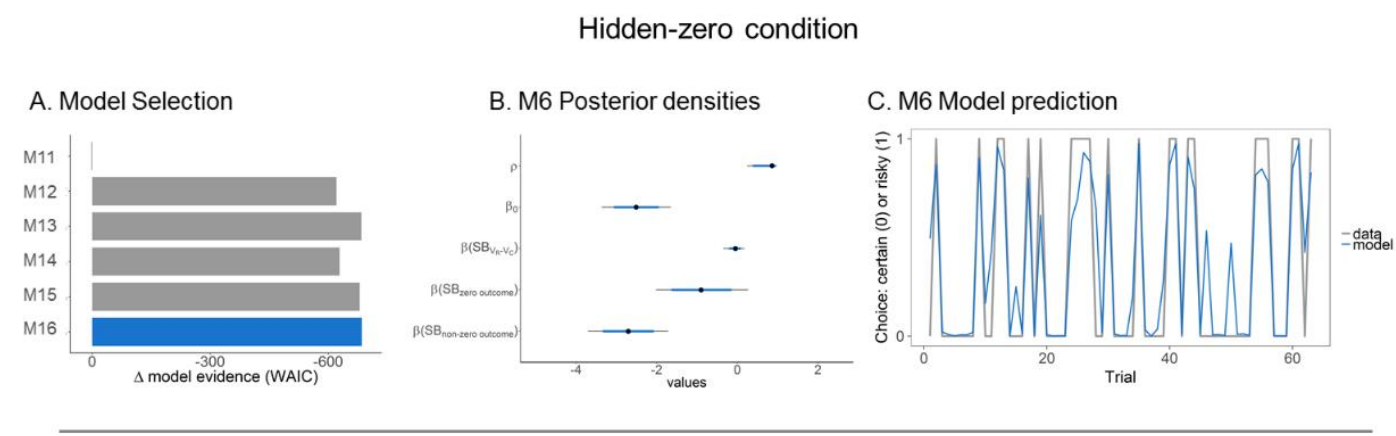

Explicit-zero condition
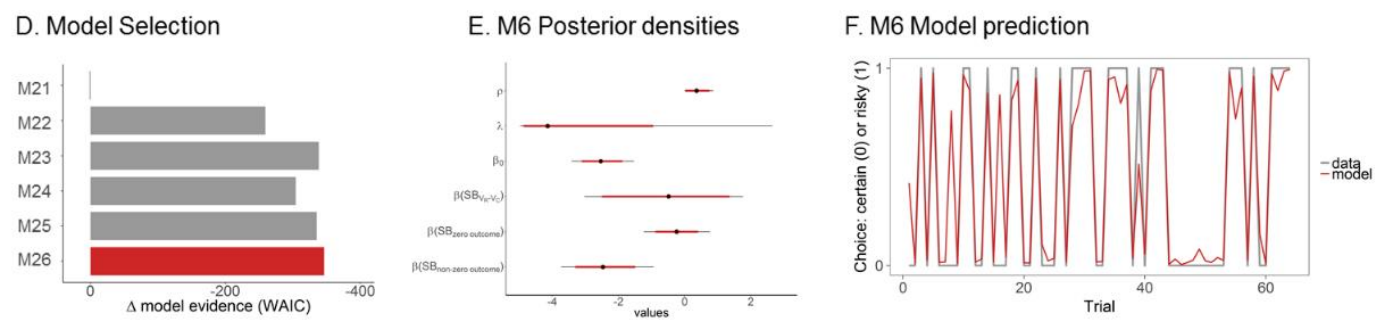

Figure 5. Model evidence and parameter inference of computational models in Study 2 .

Top panel: hidden-zero condition. (A) Model evidence (WAIC) with respect to M11 favors M16 (shown in blue). Lower WAIC (i.e., more negative) indicates better out-of-sample predictive accuracy. (B) Posterior density of the winning model M16. Blue bars show the 95\% highest density interval (HDI). (C) Posterior model prediction of the winning model M16 to verify whether the model (blue line) recapitulates the behavioral data (gray line) for an example participant. The modelgenerated prediction well matched the example participant's actual choices.

Bottom panel: explicit -zero condition. (D) Model evidence (WAIC) with respect to M21 favors M26 (shown in red). (E) Posterior density of the winning model M26. Red bars show the 95\% highest density interval (HDI). (F) Posterior model prediction of the winning model M26 to verify whether the model (red line) recapitulates the behavioral data (gray line) for an example participant. The modelgenerated prediction well matched the example participant's actual choices. 
HIDDEN-ZERO EFFECT IN RISKY DECISION-MAKING

\section{Appendix}

2 Table A1

3 Results of the generalized linear mixed model examining the effects on choice

4 preference.

\begin{tabular}{llc}
\hline Fixed effects & $\beta(S E)$ & Odds Ratio $(95 \% \mathrm{CI})$ \\
\hline Intercept & $1.84(0.25)^{* *}$ & $6.27(3.87-10.30)$ \\
Condition(hidden-/explicit-zero) & $-0.93(0.16)^{* *}$ & $0.39(0.29-0.54)$ \\
Probability & $-0.01(0.21)$ & $0.99(0.66-1.51)$ \\
Condition*Probability & $0.01(0.13)$ & $1.01(0.78-1.31)$ \\
Model fit & BIC: 10943.3 & Prediction rate $(\%)$ \\
& & $61.2 \%$ \\
\hline
\end{tabular}

5

Note. $\mathrm{BIC}=$ Bayesian information criterion.

6

${ }^{\dagger} p<0.1,{ }^{*} p<0.05,{ }^{* *} p<0.01$.

7

8 
HIDDEN-ZERO EFFECT IN RISKY DECISION-MAKING

\section{Table A2}

2 Results of the generalized linear mixed model examining the effects on choice

3 preference.

\begin{tabular}{llc}
\hline Fixed effects & $\beta(S E)$ & Odds Ratio $(95 \% \mathrm{CI})$ \\
\hline Intercept & $2.44(0.53)^{* *}$ & $11.52(4.03-34.08)$ \\
Condition(hidden-/explicit-zero) & $-0.84(0.33)^{* *}$ & $0.43(0.22-0.83)$ \\
Probability & $0.09(0.39)$ & $1.10(0.51-2.40)$ \\
Condition*Probability & $-0.82(0.25)^{* *}$ & $2.26(1.39-3.68)$ \\
Trial repeated times & $-0.11(0.05)^{*}$ & $0.90(0.82-0.99)$ \\
Model fit & BIC: 3146.80 & Prediction rate $(\%):$ \\
& & $81.52 \%$ \\
\hline
\end{tabular}

Note. RPS, risk propensity scale; $\mathrm{BIC}=$ Bayesian information criterion.

$$
{ }^{\dagger} p<0.1,{ }^{*} p<0.05,{ }^{* *} p<0.01 \text {. }
$$


HIDDEN-ZERO EFFECT IN RISKY DECISION-MAKING

Table A3

2 Results of the generalized linear mixed model examining the effects on SB scores.

\begin{tabular}{|c|c|c|c|c|c|c|}
\hline \multirow{2}{*}{ Fixed effects } & \multicolumn{2}{|c|}{$\mathrm{SB}_{\text {option }}$} & \multicolumn{2}{|c|}{$\mathrm{SB}_{\text {zero_outcome }}$} & \multicolumn{2}{|c|}{$\mathrm{SB}_{\text {non-zero_outcome }}$} \\
\hline & $\beta(S E)$ & Odds ratio $(95 \% \mathrm{CI})$ & $\beta(S E)$ & Odds ratio $(95 \% \mathrm{CI})$ & $\beta(S E)$ & Odds ratio $(95 \% \mathrm{CI})$ \\
\hline Intercept & $0.22(0.05)^{* *}$ & $1.24(1.12-1.37)$ & $0.07(0.02)^{* *}$ & $1.07(1.04-1.11)$ & $0.14(0.05)^{* *}$ & $1.15(1.05-1.26)$ \\
\hline $\begin{array}{l}\text { Condition(hidden- } \\
\text { /explicit-zero) }\end{array}$ & $-0.18(0.03)^{* *}$ & $0.84(0.79-0.89)$ & $-0.10(0.01)^{* *}$ & $0.91(0.89-0.92)$ & $-0.08(0.03)^{* *}$ & $0.92(0.87-0.98)$ \\
\hline Probability & $-0.09(0.04)^{*}$ & $0.91(0.85-0.98)$ & $-0.05(0.02)^{* *}$ & $0.95(0.92-0.98)$ & $-0.04(0.03)$ & $0.96(0.91-1.03)$ \\
\hline Condition*Probability & $0.09(0.02)^{* *}$ & $1.09(1.04-1.15)$ & $0.05(0.01)^{* *}$ & $1.06(1.03-1.08)$ & $0.03(0.02)^{\dagger}$ & $1.04(0.99-1.08)$ \\
\hline Trial repeated times & $0.03(0.01)^{* *}$ & $1.03(1.02-1.04)$ & $0.02(0.003)^{* *}$ & $1.02(1.01-1.02)$ & $0.01(0.004)^{*}$ & $1.01(1.00-1.02)$ \\
\hline Model fit & BIC : 1351.60 & MSE: 0.08 & BIC : -3591.20 & MSE: 0.02 & $\mathrm{BIC}: 63.40$ & MSE: 00.06 \\
\hline
\end{tabular}

Note. RPS, risk propensity scale; $\mathrm{BIC}=$ Bayesian information criterion; $\mathrm{MSE}=$ mean square error

$$
{ }^{\dagger} p<0.1{ }^{*} p<0.05,{ }^{* *} p<0.01 \text {. }
$$


HIDDEN-ZERO EFFECT IN RISKY DECISION-MAKING

Experimental pairs of options in explicit-zero condition from Study 1.

\begin{tabular}{|c|c|c|c|c|c|c|c|c|}
\hline \multirow{3}{*}{$\begin{array}{c}\text { Trial } \\
\text { number }\end{array}$} & \multicolumn{4}{|c|}{ Risky option } & \multicolumn{4}{|c|}{ Certain option } \\
\hline & \multicolumn{2}{|c|}{ Non-zero outcome } & \multicolumn{2}{|c|}{$\begin{array}{c}\text { Zero } \\
\text { outcome }\end{array}$} & \multicolumn{2}{|c|}{ Non-zero outcome } & \multicolumn{2}{|c|}{ Zero outcome } \\
\hline & $\%$ & payoff & $\%$ & payoff & $\%$ & payoff & $\%$ & payoff \\
\hline 1 & $30 \%$ & 309 & $70 \%$ & 0 & $100 \%$ & 92 & $0 \%$ & 0 \\
\hline 2 & $91 \%$ & 99 & $9 \%$ & 0 & $100 \%$ & 90 & $0 \%$ & 0 \\
\hline 3 & $75 \%$ & 127 & $25 \%$ & 0 & $100 \%$ & 95 & $0 \%$ & 0 \\
\hline 4 & $92 \%$ & 99 & $8 \%$ & 0 & $100 \%$ & 91 & $0 \%$ & 0 \\
\hline 5 & $71 \%$ & 136 & $29 \%$ & 0 & $100 \%$ & 96 & $0 \%$ & 0 \\
\hline 6 & $26 \%$ & 379 & $74 \%$ & 0 & $100 \%$ & 97 & $0 \%$ & 0 \\
\hline 7 & $90 \%$ & 110 & $10 \%$ & 0 & $100 \%$ & 99 & $0 \%$ & 0 \\
\hline 8 & $88 \%$ & 106 & $12 \%$ & 0 & $100 \%$ & 93 & $0 \%$ & 0 \\
\hline 9 & $79 \%$ & 116 & $21 \%$ & 0 & $100 \%$ & 92 & $0 \%$ & 0 \\
\hline 10 & $12 \%$ & 840 & $88 \%$ & 0 & $100 \%$ & 98 & $0 \%$ & 0 \\
\hline 11 & $82 \%$ & 112 & $18 \%$ & 0 & $100 \%$ & 92 & $0 \%$ & 0 \\
\hline 12 & $24 \%$ & 405 & $76 \%$ & 0 & $100 \%$ & 98 & $0 \%$ & 0 \\
\hline 13 & $88 \%$ & 109 & $12 \%$ & 0 & $100 \%$ & 96 & $0 \%$ & 0 \\
\hline 14 & $84 \%$ & 113 & $16 \%$ & 0 & $100 \%$ & 96 & $0 \%$ & 0 \\
\hline 15 & $82 \%$ & 120 & $18 \%$ & 0 & $100 \%$ & 99 & $0 \%$ & 0 \\
\hline 16 & $5 \%$ & 1828 & $95 \%$ & 0 & $100 \%$ & 95 & $0 \%$ & 0 \\
\hline 17 & $15 \%$ & 619 & $85 \%$ & 0 & $100 \%$ & 94 & $0 \%$ & 0 \\
\hline 18 & $28 \%$ & 340 & $72 \%$ & 0 & $100 \%$ & 95 & $0 \%$ & 0 \\
\hline 19 & $76 \%$ & 130 & $24 \%$ & 0 & $100 \%$ & 99 & $0 \%$ & 0 \\
\hline 20 & $7 \%$ & 1343 & $93 \%$ & 0 & $100 \%$ & 92 & $0 \%$ & 0 \\
\hline 21 & $27 \%$ & 336 & $73 \%$ & 0 & $100 \%$ & 90 & $0 \%$ & 0 \\
\hline 22 & $86 \%$ & 115 & $14 \%$ & 0 & $100 \%$ & 99 & $0 \%$ & 0 \\
\hline 23 & $23 \%$ & 400 & $77 \%$ & 0 & $100 \%$ & 90 & $0 \%$ & 0 \\
\hline 24 & $78 \%$ & 123 & $22 \%$ & 0 & $100 \%$ & 95 & $0 \%$ & 0 \\
\hline
\end{tabular}


HIDDEN-ZERO EFFECT IN RISKY DECISION-MAKING

\begin{tabular}{|c|c|c|c|c|c|c|c|c|}
\hline 25 & $10 \%$ & 952 & $90 \%$ & 0 & $100 \%$ & 94 & $0 \%$ & 0 \\
\hline 26 & $22 \%$ & 411 & $78 \%$ & 0 & $100 \%$ & 92 & $0 \%$ & 0 \\
\hline 27 & $93 \%$ & 106 & $7 \%$ & 0 & $100 \%$ & 98 & $0 \%$ & 0 \\
\hline 28 & $15 \%$ & 634 & $85 \%$ & 0 & $100 \%$ & 96 & $0 \%$ & 0 \\
\hline 29 & $26 \%$ & 371 & $74 \%$ & 0 & $100 \%$ & 98 & $0 \%$ & 0 \\
\hline 30 & $5 \%$ & 1730 & $95 \%$ & 0 & $100 \%$ & 92 & $0 \%$ & 0 \\
\hline 31 & $27 \%$ & 369 & $73 \%$ & 0 & $100 \%$ & 98 & $0 \%$ & 0 \\
\hline 32 & $93 \%$ & 101 & $7 \%$ & 0 & $100 \%$ & 94 & $0 \%$ & 0 \\
\hline 33 & $11 \%$ & 834 & $89 \%$ & 0 & $100 \%$ & 95 & $0 \%$ & 0 \\
\hline 34 & $93 \%$ & 100 & $7 \%$ & 0 & $100 \%$ & 93 & $0 \%$ & 0 \\
\hline 35 & $89 \%$ & 109 & $11 \%$ & 0 & $100 \%$ & 97 & $0 \%$ & 0 \\
\hline 36 & $27 \%$ & 351 & $73 \%$ & 0 & $100 \%$ & 95 & $0 \%$ & 0 \\
\hline 37 & $70 \%$ & 140 & $30 \%$ & 0 & $100 \%$ & 99 & $0 \%$ & 0 \\
\hline 38 & $89 \%$ & 107 & $11 \%$ & 0 & $100 \%$ & 96 & $0 \%$ & 0 \\
\hline 39 & $94 \%$ & 105 & $6 \%$ & 0 & $100 \%$ & 99 & $0 \%$ & 0 \\
\hline 40 & $28 \%$ & 357 & $72 \%$ & 0 & $100 \%$ & 99 & $0 \%$ & 0 \\
\hline 41 & $28 \%$ & 322 & $72 \%$ & 0 & $100 \%$ & 92 & $0 \%$ & 0 \\
\hline 42 & $7 \%$ & 1386 & $93 \%$ & 0 & $100 \%$ & 93 & $0 \%$ & 0 \\
\hline 43 & $83 \%$ & 113 & $17 \%$ & 0 & $100 \%$ & 94 & $0 \%$ & 0 \\
\hline 44 & $10 \%$ & 901 & $90 \%$ & 0 & $100 \%$ & 91 & $0 \%$ & 0 \\
\hline 45 & $91 \%$ & 107 & $9 \%$ & 0 & $100 \%$ & 97 & $0 \%$ & 0 \\
\hline 46 & $5 \%$ & 1784 & $95 \%$ & 0 & $100 \%$ & 93 & $0 \%$ & 0 \\
\hline 47 & $78 \%$ & 123 & $22 \%$ & 0 & $100 \%$ & 95 & $0 \%$ & 0 \\
\hline 48 & $88 \%$ & 105 & $12 \%$ & 0 & $100 \%$ & 93 & $0 \%$ & 0 \\
\hline 49 & $92 \%$ & 102 & $8 \%$ & 0 & $100 \%$ & 94 & $0 \%$ & 0 \\
\hline 50 & $77 \%$ & 117 & $23 \%$ & 0 & $100 \%$ & 90 & $0 \%$ & 0 \\
\hline 51 & $90 \%$ & 100 & $10 \%$ & 0 & $100 \%$ & 90 & $0 \%$ & 0 \\
\hline 52 & $7 \%$ & 1305 & $93 \%$ & 0 & $100 \%$ & 95 & $0 \%$ & 0 \\
\hline 53 & $79 \%$ & 120 & $21 \%$ & 0 & $100 \%$ & 94 & $0 \%$ & 0 \\
\hline
\end{tabular}


HIDDEN-ZERO EFFECT IN RISKY DECISION-MAKING

\begin{tabular}{lcccc|cccc}
\hline 54 & $21 \%$ & 443 & $79 \%$ & 0 & $100 \%$ & 95 & $0 \%$ & 0 \\
55 & $20 \%$ & 491 & $80 \%$ & 0 & $100 \%$ & 98 & $0 \%$ & 0 \\
56 & $5 \%$ & 1841 & $95 \%$ & 0 & $100 \%$ & 96 & $0 \%$ & 0 \\
57 & $79 \%$ & 122 & $21 \%$ & 0 & $100 \%$ & 97 & $0 \%$ & 0 \\
58 & $29 \%$ & 327 & $71 \%$ & 0 & $100 \%$ & 94 & $0 \%$ & 0 \\
59 & $78 \%$ & 117 & $22 \%$ & 0 & $100 \%$ & 91 & $0 \%$ & 0 \\
60 & $10 \%$ & 944 & $90 \%$ & 0 & $100 \%$ & 93 & $0 \%$ & 0 \\
61 & $92 \%$ & 102 & $8 \%$ & 0 & $100 \%$ & 94 & $0 \%$ & 0 \\
62 & $5 \%$ & 1798 & $95 \%$ & 0 & $100 \%$ & 92 & $0 \%$ & 0 \\
63 & $19 \%$ & 489 & $81 \%$ & 0 & $100 \%$ & 90 & $0 \%$ & 0 \\
\hline
\end{tabular}

Note. payoff indicates the value of the corresponding payoffs, and the $\%$

2 indicates the probability of the corresponding payoff. The option pairs were the same

3 in the hidden-zero condition, except for the zero outcomes. 
HIDDEN-ZERO EFFECT IN RISKY DECISION-MAKING

Table A5

Experimental pairs of options in the explicit-zero condition from Study 2.

\begin{tabular}{ccccc|cccc}
\hline \multirow{2}{*}{$\begin{array}{c}\text { Trial } \\
\text { number }\end{array}$} & \multicolumn{3}{c|}{ Risky option } & \multicolumn{3}{c}{ Certain option } \\
\cline { 2 - 9 } & Non-zero outcome & \multicolumn{2}{c}{ Zero } & Non-zero outcome & Zero outcome \\
\cline { 2 - 9 } & $\%$ & payoff & $\%$ & payoff & $\%$ & payoff & $\%$ & payoff \\
\hline 1 & $94 \%$ & 104 & $6 \%$ & 0 & $100 \%$ & 98 & $0 \%$ & 0 \\
2 & $89 \%$ & 106 & $11 \%$ & 0 & $100 \%$ & 95 & $0 \%$ & 0 \\
3 & $84 \%$ & 115 & $16 \%$ & 0 & $100 \%$ & 97 & $0 \%$ & 0 \\
4 & $7 \%$ & 1342 & $93 \%$ & 0 & $100 \%$ & 94 & $0 \%$ & 0 \\
5 & $9 \%$ & 1066 & $91 \%$ & 0 & $100 \%$ & 96 & $0 \%$ & 0 \\
6 & $8 \%$ & 1187 & $92 \%$ & 0 & $100 \%$ & 95 & $0 \%$ & 0 \\
7 & $15 \%$ & 633 & $85 \%$ & 0 & $100 \%$ & 95 & $0 \%$ & 0 \\
8 & $95 \%$ & 95 & $5 \%$ & 0 & $100 \%$ & 91 & $0 \%$ & 0 \\
9 & $83 \%$ & 115 & $17 \%$ & 0 & $100 \%$ & 96 & $0 \%$ & 0 \\
10 & $91 \%$ & 106 & $9 \%$ & 0 & $100 \%$ & 97 & $0 \%$ & 0 \\
11 & $82 \%$ & 112 & $18 \%$ & 0 & $100 \%$ & 92 & $0 \%$ & 0 \\
12 & $92 \%$ & 102 & $8 \%$ & 0 & $100 \%$ & 94 & $0 \%$ & 0 \\
13 & $11 \%$ & 863 & $89 \%$ & 0 & $100 \%$ & 95 & $0 \%$ & 0 \\
14 & $20 \%$ & 478 & $80 \%$ & 0 & $100 \%$ & 98 & $0 \%$ & 0 \\
15 & $5 \%$ & 1848 & $95 \%$ & 0 & $100 \%$ & 93 & $0 \%$ & 0 \\
\hline & $19 \%$ & 484 & $81 \%$ & 0 & $100 \%$ & 92 & $0 \%$ & 0 \\
\hline
\end{tabular}

Note. payoff indicates the value of the corresponding payoffs, and the $\%$

4 indicates the probability of the corresponding payoff. The option pairs were the same

5 in the hidden-zero condition, except for the zero outcomes. All the option pairs were

6 repeated a fourth time with a different presentation mode across blocks. 
HIDDEN-ZERO EFFECT IN RISKY DECISION-MAKING

1 Table A6

2 Summary of candidate computational models in Study 1 and Study 2.

3

\begin{tabular}{|c|c|c|c|}
\hline Condition & Study & Candidate Model & Description \\
\hline \multirow{7}{*}{ Hidden-zero } & \multirow{2}{*}{ Study $1 / 2$} & M11 & RA \\
\hline & & M12 & aRA \\
\hline & \multirow{5}{*}{ Study 2} & M13 & $\mathrm{aRA}+\mathrm{SB}_{\text {option }}$ \\
\hline & & M14 & $\mathrm{aRA}+\mathrm{SB}_{\text {zero outcome }}$ \\
\hline & & & \\
\hline & & M15 & aRA $+\mathrm{SB}_{\text {non-zero_outcome }}$ \\
\hline & & M16 & $\mathrm{aRA}+\mathrm{SB}_{\text {zero_outcome }}+\mathrm{SB}_{\text {non-zero_outcome }}$ \\
\hline \multirow{7}{*}{ Explicit-zero } & \multirow{2}{*}{ Study $1 / 2$} & M21 & $\mathrm{RA}-p \lambda$ \\
\hline & & M22 & aRA-p $\lambda$ \\
\hline & \multirow{5}{*}{ Study 2} & M23 & aRA-p $\lambda+\mathrm{SB}_{\text {option }}$ \\
\hline & & M24 & aRA- $p \lambda+\mathrm{SB}_{\text {zero outcome }}$ \\
\hline & & & \\
\hline & & M25 & aRA-p $p \lambda+\mathrm{SB}_{\text {non-zero_outcome }}$ \\
\hline & & M26 & aRA-p $p \lambda+\mathrm{SB}_{\text {zero_outcome + }} \mathrm{SB}_{\text {non-zero_outcome }}$ \\
\hline
\end{tabular}

4 Note. $\mathrm{RA}=$ risk-aversion model; $\mathrm{aRA}=$ attentional risk-aversion model; $\mathrm{SB}=$ 5 attentional bias scores computed from the eye-movement data.

6 